Estudios Constitucionales, Año 12, No 1, 2014, pp. 589-593.

ISSN 07180195

Centro de Estudios Constitucionales de Chile Universidad de Talca

Latin American Constitutionalism, 1810-2010. The Engine Room

of the Constitution. Roberto Gargarella

Jaime Gajardo Falcón

\title{
LATIN AMERICAN CONSTITUTIONALISM, 1810-2010. THE ENGINE ROOM OF THE CONSTITUTION
}

Roberto Gargarella

Oxford University Press, Oxford, 2013, 283 Pp.

\author{
Jaime Gajardo Falcón* \\ Universidad Autónoma de Madrid - España \\ gajardofalcon@gmail.com
}

La obra del jurista y sociólogo argentino destaca por la importancia de la temática abordada y resulta relevante para la doctrina constitucional latinoamericana. Junto con sintetizar las ideas políticas, filosóficas y jurídicas plasmadas en sus investigaciones de las últimas dos décadas, con la obra recoge la riqueza de doscientos años de constitucionalismo latinoamericano y problematiza sobre su estado actual y perspectivas de desarrollo ${ }^{1}$.

El autor nos señala que el libro se origina por una doble disconformidad intelectual. En primer lugar, por el hecho de que el constitucionalismo latinoamericano no ha sido objeto de una atención académica sistemática y pública, al menos hasta hace poco, a pesar de su larga y rica historia. En segundo lugar, por la forma en que el constitucionalismo regional ha afrontado las reformas constitucionales (sobre todo en las últimas décadas), poniendo una obsesiva atención a los derechos constitucionales, en detrimento de la organización del poder. Según el autor, ello ha dado como resultado que el sistema concentrado de poder entre en conflicto con las demandas sociales que se generan en nombre de los nuevos derechos constitucionales y, por ende, que una parte de la Constitución (orgánica) comience a trabajar en contra del éxito de la segunda (dogmática).

\footnotetext{
* Abogado, Licenciado en Ciencias Jurídicas y Sociales, Universidad de Chile. Magíster en Derecho, con mención en Derecho Público, Universidad de Chile. Máster en Gobernanza y Derechos Humanos, Universidad Autónoma de Madrid. Máster en Derecho Constitucional, Centro de Estudios Políticos y Constitucionales (España). Doctorando en Derecho y Ciencia Política, Universidad Autónoma de Madrid. Personal Investigador en Formación, Universidad Autónoma de Madrid.

${ }^{1}$ Relacionada con esta temática, véase la siguiente obra del autor: The Legal Foundations of Inequality: Constitutionalism in the Americas, 1776-1860 (Cambridge, Cambridge University Press, 2010).
} 
Así, el libro tiene por objeto de estudio, el análisis de doscientos años de constitucionalismo latinoamericano (1810-2010), con el propósito de describir lo que ha sucedido en el campo a lo largo de los años y, a partir de ahí, extraer la teoría constitucional. Junto con tomarse en serio la teoría constitucional latinoamericana, analiza el pensamiento de importantes figuras políticas, intelectuales y teóricos del Derecho, tales como: Juan Bautista Alberdi, Francisco Bilbao, Simón Bolívar, Juan Egaña, Andrés Bello, José González Vigil, Victorino Lastarria, Juan Carlos Mariátegui, Juan Montalvo, José María Mora, Mariano Otero, Manuel Murillo Toro, José María Samper y Domingo Sarmiento.

El propósito del libro es descriptivo y normativo. Descriptivamente, al revisar brevemente doscientos años de constitucionalismo. Normativamente, al repensar el constitucionalismo regional desde una perspectiva igualitaria.

La obra está marcada por el hecho social de la desigualdad latinoamericana, que configura las diferentes esferas jurídicas y proyectos constitucionales, afectando histórica y sistemáticamente a ciertos grupos sociales. La idea principal de la obra es que el sistema institucional tiene una responsabilidad importante en la consolidación del sistema político, económico y social, que sigue siendo, después de doscientos años de independencia, profundamente desigual.

En su análisis histórico, la obra se organiza en torno a cinco períodos claves. La primera fase constitucional se extiende desde la independencia hasta la mitad del siglo XIX (Capítulo 1). Siguiendo la pretensión entregada por Juan Bautista Alberdi sobre la función del constitucionalismo, el autor analiza el trabajo constitucional de Simón Bolivar y Juan Egaña, buscando la respuesta que entregan sus proyectos constitucionales a los "dramas" de la época. Sintetiza el conflicto constitucional, en dos ideas fundacionales (autonomía individual y autogobierno colectivo) y tres modelos constitucionales (conservador, liberal y republicano o radical) que las abordan.

El segundo período se inicia a mediados del siglo XIX y continúa hasta comienzos del siglo XX (Capítulos 2, 3 y 4). El autor señala que este es el período "fundacional" del constitucionalismo latinoamericano, en el cual se escribieron las principales constituciones y cuya influencia se siente hasta el día de hoy. Este será el período de "orden y progreso" y de consolidación de la estructura legal poscolonial. Aquí da cuenta de las discusiones que se dieron en torno a la herencia del pasado, la tesis de la "independencia" y su impacto en la restricción de los derechos políticos (sufragio). Ocupa un lugar preponderante, en el análisis del autor, la alianza liberal-conservadora y sus consecuencias en términos constitucionales. 
El tercer período corresponde a la crisis del constitucionalismo poscolonial (Capítulo 5), que va de finales del siglo XIX a principios del XX, marcado por la presencia de las ideas positivistas. Representa el momento de ruptura con el orden legal poscolonial y el surgimiento de alternativas al modelo, tales como la tendencia radical agraria (agrarismo, indigenismo, socialismo), la democrática (Argentina y Uruguay) y la revolucionaria (México), la que es especialmente analizada por el autor, por su enorme influencia en Latinoamérica. Además, el autor realiza un profundo análisis a la idea de politics of presence ${ }^{2}$ en la Convención constitucional mexicana de Aguascaliente, donde participaron grupos sociales y políticos que habían sido excluidos de la participación política, resultando fundamental para la inclusión de la "cuestión social" en la Constitución mexicana de 1917.

La cuarta fase es la del constitucionalismo social (Capítulos 6 y 7), que se inicia con la crisis económica de 1930 y tiene su punto cúlmine a mediados del siglo XX. Aparecen numerosos proyectos constitucionales, cuyo objetivo era recuperar la "cuestión social" que las viejas constituciones del siglo XIX habían olvidado o descuidado. Es el período de término del orden neocolonial, por lo cual el autor centra su atención en las respuestas constitucionales y políticas que ofrece el nuevo siglo, donde el surgimiento de los derechos sociales representa la novedad más significativa. En este punto, Gargarella es crítico con la introducción de los derechos sociales a las constituciones y escéptico de sus potenciales virtudes. Problematiza, sutil y meticulosamente, el impacto que causan los derechos sociales en constituciones que son hostiles con las demandas que crean dichos derechos. Asimismo, no le convence la defensa teórica que han tenido los derechos sociales por parte de la izquierda y apunta las contradicciones que habría en ello.

El período de la quinta y última fase se extiende desde finales del siglo XX hasta el inicio del siglo actual (Capítulos 8 y 9). El autor se centra en el "nuevo constitucionalismo latinoamericano", que, en términos generales, expande los compromisos sociales de las constituciones anteriores, pero mantiene el viejo modelo de concentración del poder.

Inicia el análisis del constitucionalismo latinoamericano contemporáneo, revisando las constituciones que se encuentran en tensión interna (Capítulo 9), esto es, aquellas nacidas como reacciones directas al autoritarismo de la década de 1970, las que establecen el escenario para la aplicación de los programas de "ajuste estructural" durante la década de 1990 (Brasil, Colombia, Perú y Argentina), y,

2 Véase Kymlicka, Will (1995): The Rights of Minority Cultures (Oxford, Oxford University Press); PHiLlips, Anne (1995): The Politics of Presence (Oxford, Oxford University Press). 
por último, las que fueron reacciones a la grave crisis social provocada por los programas "neoliberales".

Luego, dedica unas breves páginas para abordar la extraordinaria transformación que ha supuesto la apertura del constitucionalismo latinoamericano al Derecho Internacional de los Derechos Humanos y, en particular, al Sistema Interamericano de Protección de los Derechos Humanos. Junto con tomar nota del fenómeno, presenta argumentos críticos, principalmente desde el déficit democrático del sistema, señalando como ejemplo paradigmático de ello la sentencia de la Corte Interamericana de Derechos Humanos en el caso Gelman vs. Uruguay (2011).

Continúa con el análisis del constitucionalismo contemporáneo (Capítulo 10), ilustrando la dinámica de centralización del poder frente a la expansión de los derechos, con algunos ejemplos procedentes del nuevo constitucionalismo latinoamericano (Ecuador, Venezuela y México). En particular, centra su atención en el ejemplo de la introducción de los derechos indígenas en las nuevas constituciones (utiliza el ejemplo de Bolivia como paradigma) y en las dificultades (o falta de voluntad política) para entrar en la "sala de máquinas" de la Constitución y modificar la estructura de organización del poder. Explora algunos "modestos" intentos dirigidos a la introducción de reformas en la "sala de máquinas", dentro de ellos, la Sala IV de la Corte Suprema de Costa Rica y la Corte Constitucional colombiana creada por la Constitución de 1991. El ejemplo de estas reformas le sirve para ilustrar que la entrada en la "sala de máquinas" del constitucionalismo puede reconocer diferentes formas y búsquedas.

Concluye el libro (Capítulo 10) preguntándose: ¿qué hemos aprendido en 200 años de constitucionalismo? Resumiendo la primera parte del libro, señala que al contestar la pregunta de Alberdi sobre cuál es el "drama" social del cual la Constitución se debe hacer cargo, constata la existencia de una demanda por autogobierno colectivo y autonomía individual, que no ha podido ser honrada de forma conjunta por el constitucionalismo latinoamericano. Para el autor, el ejercicio histórico tiene una utilidad descriptiva-normativa, ya que nos permite conocer qué es lo que debemos considerar al momento de realizar reformas legalesconstitucionales que busquen resolver los grandes "dramas" de Latinoamérica. En ese sentido, apunta seis aspectos que considera claves, a saber: 1) La necesidad de consistencia externa e interna de las reformas; 2) El cuidado que se debe tener con la ambición en la radicalidad de las reformas y su relación con la limitación de la racionalidad 3 ; 3) Enfatiza la idea de presence y sus beneficios en las reformas

3 Véase ELSTER, Jon (1989): Salomonic Judgements (Cambridge, Cambridge University Press). 
constitucionales; 4) Recoge las virtudes de las reformas localizadas y sus impactos; 5) La necesidad de reformas en la "sala de máquinas" de la Constitución; 6) La importancia de trabajar sobre las bases materiales de la Constitución.

Latin American Constitutionalism es un libro importante para la doctrina constitucional latinoamericana. Combina el análisis socio-político, histórico, filosófico y jurídico, proporcionándonos un gran trabajo de síntesis y una atractiva propuesta teórica para analizar el devenir del constitucionalismo regional. Asimismo, considerando la coyuntura constitucional en la que se encuentra inmerso Chile, la monografía nos permite distinguir las luces y sombras del constitucionalismo latinoamericano y los aspectos que se deben tener en cuenta para la reforma de la Constitución desde una perspectiva igualitaria. 